\title{
La guerre et l'évitement de la loi dans Jules César et dans Macbeth
}

\section{Daniel Sibony}

\section{(2) OpenEdition}

1 Journals

\section{Édition électronique}

URL : http://journals.openedition.org/shakespeare/1219

DOI : 10.4000/shakespeare.1219

ISSN : 2271-6424

Éditeur

Société Française Shakespeare

\section{Édition imprimée}

Date de publication : 1 novembre 1990

Pagination : 129-138

Référence électronique

Daniel Sibony, "La guerre et l'évitement de la loi dans Jules César et dans Macbeth », Actes des congrès de la Société française Shakespeare [En ligne], 8| 1990, mis en ligne le 01 janvier 2007, consulté le 06 mai 2019. URL : http://journals.openedition.org/shakespeare/1219; DOI : 10.4000/shakespeare.1219 


\section{SHAKESPEARE \\ ET LA GUERRE}

Ouvrage publié avec le concours de la Commission

des Communautés Européennes 
Illustration de la couverture : DERRICKE'S Image of Ireland, 1586 


\section{La Guerre et l'Evitement de la Loi dans Jules César et dans Macbeth}

Quand j'ai donné ce titre l'an dernier je venais de terminer le premier tome d'Avec Shakespeare et j'étais encore sous le coup de l'émotion de ma fréquentation d'une pièce aussi gigantesque que Jules César, et en partie aussi Macbeth. Aujourd'hui je vais déborder ce titre car cette année j'ai commencé le second cycle Shakespeare de mon séminaire avec les Henri VI dont j'ai parlé mardi dernier.

Tout d'abord, qu'est-ce que c'est que la guerre? On ne peut pas se contenter d'un point de vue élémentaire qui veut que ce soit l'affrontement de deux camps qui échouent à se départager, et qui en viennent aux mains dans ce duel. Je pense que Shakespeare a toujours des dimensions, des dynamiques beaucoup plus riches pour explorer ce phénomène étonnant. Et puisque, $\mathrm{j}$ 'imagine, l'étymologie latine vous est connue, je vous en proposerai une autre, biblique, puisque Shakespeare était grand lecteur de la Bible. La guerre se dit d'un mot qui dans les langues sémitiques se réfère au pain, à la viande. C'est la boucherie, en somme, du corps-à-corps. Mais ces mises en acte du corps, à la fois recours ultime - puisque la parole est impuissante à décider, et que la Loi s'est fracassée ou se révèle vacillante - ces mises en jeu du corps sont en même temps un recours ultime au réel pris comme le lieu de la vérité, d'où l'on attend que le destin s'exprime; le destin, la divinité, bref l'ensemble des forces qui explicitent nos désirs, nos potentiels cachés, et qui bien sûr les dépassent. De sorte que c'est souvent après-coup qu'une guerre apparaît bonne ou juste ou injuste. Certes, on ne 
sait pas où s'arrête l'après-coup; il est souvent suivi d'un autre; l'instance qui compte ou qui inscrit les après-coups est en partie ouverte. Au départ, il s'agit de savoir interroger assez bien le destin pour que la guerre, en cours ou en jeu, puisse trouver sa «justesse», en tant qu'événement d'une loi ébranlée qui tente de rétablir le lien avec la loi symbolique. De sorte qu'on assiste, dans la Bible, à des répliques du genre: «tu peux y aller», «Dieu les a donnés entre tes mains, tes ennemis», «Dieu te les a livrés». Autrement dit, la guerre n'est pas seulement un affrontement de deux termes, c'est une entreprise d'accouchement douloureux d'une certaine vérité du collectif qui est en proie à cette génèse, à cette douleur de gestation; et c'est un collectif multiple, déchiré, qui essaie dans la guerre - c'est pourquoi c'est violent - d'affronter, voire d'accueillir en lui cette altérité qui s'appelle la Loi, et qui dépasse les lois écrites. La Loi, en l'occurrence, serait une certaine proximité à ce point chaud et lumineux qu'on peut appeler la souveraineté (qui est différente du pouvoir). Avoir le pouvoir - souvent c'est ne pas pouvoir grand'chose - avoir le pouvoir c'est plus fermé qu'avoir la souveraineté; celle-ci est un lieu qui a été agréé et même aimé par le peuple, par le collectif. De ce point de vue, ce qui se passe dans Jules César est passionnant. Il y a là un souverain, Jules César, dont on s'aperçoit très vite qu'il est foutu parce qu'il se prend pour Jules César; et ça, ça ne pardonne pas, de se prendre pour ce qu'on est, surtout si ce qu'on est est tellement proche de cette place fragile, celle de la souveraineté : où le roi est au-dessus du peuple mais en même temps c'est par le peuple qu'il est roi. Autrement dit, s'il y a une place où chacun - parce que le roi c'est un peu chacun - (ainsi les choses complexes prennent dans Shakespeare une étonnante simplicité), donc s'il y a une place où tout un chacun et tout roi doit faire preuve d'une certaine distance à soi-même c'est bien la place de la souveraineté.

Donc, Jules César est déjà sur le déclin par saturation narcissique ; et voilà que sa souveraineté, bien qu'approuvée par le peuple, déplaît à Brutus et à d'autres, qui 
décident de le détruire à cette place de souveraineté. Le plus remarquable c'est qu'ils font leur complot comme une réunion de chirurgiens qui ferait son diagnostic sur un organisme malade et qui dirait: «il faut vraiment opérer...», et ils opèrent; le travail est plutôt bien fait, ablation de la tête; c'est presque dans ces termes. On a l'impression qu'ils n'ont aucun sens de la dimension symbolique du matériau qu'ils touchent; qui pourtant est surchargé; haute tension symbolique due à la souveraineté. La preuve qu'ils n'y sont pas est ce détail très théâtral, purement dramatique: une fois l'acte accompli, ces messieurs rentrent chez eux, tout simplement, et laissent à Antoine le soin de parler. Justement il en avait très envie. Mais pour eux, puisque la vérité a été accomplie, l'essentiel était fait.

En laissant sur ce mode la parole à Antoine, ils témoignent d'un mépris absolu pour la parole vive, pour la vérité changeante, et pour le peuple réel. La vérité telle que eux la voient, a quelque chose de meurtrier. Ce n'est pas bien sûr ce que pense Shakespeare, pour qui la vérité est un flux et un reflux de choses vivantes qui font vivre les humains. Et puis, on ne va pas impunément, par amour pour la république et le peuple, tuer quelqu'un qui était très aimé du peuple. On est appelé, requis, de s'en expliquer devant le peuple qui vous pose la question banale, mais radicale: «pourquoi avez-vous fait ça? pour nous? vous nous aimez donc un petit peu? eh bien restez avec nous; d'ailleurs vous êtes tellement formidable que vous méritez de remplacer Jules César. » «Surtout pas!» s'écrie Brutus, non sans pédanterie. «Pas de ça! on n'a pas enlevé un César pour en mettre un autre! » Il a sa lourde ritournelle sur la vérité qui doit s'accomplir, et sur le fait que c'est à eux de décider, etc... Reste Antoine qui, lui, va honorer cette dimension symbolique; et déjà celle du corps du souverain: il va d'abord les faire pleurer sur ce corps mort - ce qui est bien le minimum. Il les fait pleurer non pas tant sur le roi (le peuple n'est pas tellement dupe) que sur ce qui amène le peuple à se soumettre à un roi, à un maître. Et puisqu'on va changer de Maître, il faut bien 
honorer le lien antérieur; il faut bien le pleurer pour que le lien ait une valeur au moment où l'on assiste à un métabolisme du lien. À ce moment-clé, le peuple ne reçoit de Brutus et de son camp qu'une sorte de claque, d'insulte, qui accule le peuple et le confronte à sa pure et simple soumission. C'est comme si on lui disait: «vous êtes des moins que rien; et pour vous soumettre à quelqu'un il faut que vous soyez très peu mûrs» etc. C'est la ritournelle de la toute-vérité, qui est assez insupportable, et qui même en devient fausse, tant elle est saturée de vérité.

Du coup, on assiste, et là j'en viens à notre sujet, à un premier renversement: l'acte de libération que le peuple aurait pu faire pour se dégager de Jules César, il va l'accomplir sur ses «libérateurs»; il va leur faire la peau, pour rétablir un lien qui tienne, et pour retrouver une certaine dignité. C'est la guerre civile, la manifestation de masse, le déferlement contre tout ce qui touche aux conjurés. Puis on va assister, en un second temps, à l'élaboration de ce qui fait apparaître le lieu de la souveraineté comme un vide, une béance horrible. Le peuple peut changer de maître ou de souverain comme on change de chemise, ça ne prouve pas qu'il est versatile, comme le croient Cassius ou Brutus; ça prouve qu'il investit d'amour pour luimême cette place de souveraineté: autrement dit, le peuple aime son souverain parce que du lieu de la souveraineté reflue vers le peuple un certain amour pour luimême, une certaine consistance. Le peuple est prêt à changer de souverain, mais pas à laisser cette place béante et désertée au nom d'une vérité abstraite. De sorte qu'on assiste à un processus violent - dans une gradation de violence différenciée - pour accoucher d'une souveraineté tenable, vivable. Il y a un mouvement de justesse qui anime un certain camp et qui est absent de l'autre camp. Brutus et les «libérateurs», une fois qu'ils ont pris les armes, qu'ils se sont fait une armée, n'ont derrière eux plus aucune réserve symbolique; ils n'ont plus rien dont ils puissent se réclamer. Ils ont libéré le peuple, mais le peuple est blessé de cette libération qui le laisse seul.

Il y a une pièce, Lorenzaccio qui à côté est toute 
petite - Musset n'est pas Shakespeare, ce n'est pas vraiment la même échelle - mais qui développe très bien ce petit aspect politique : Lorenzaccio fait tout un cinéma, il simule la complicité avec le tyran au point de ressembler à ce qu'il simule, et il arrive à tuer le tyran; après quoi il dit au peuple: «vous gémissiez sous la tyrannie? eh bien voilà la liberté, je vous la donne; à vous de jouer. Et que fait le peuple? première mesure urgente: il lynche le libérateur. Non sans raison, car encore une fois un peuple est un organisme vivant, humain, qui ne peut pas supporter de recevoir une claque comme ça, comme un individu, et de recevoir cette claque d'un individu qui lui dit seulement son mépris et son amour de la vérité fétichisée.

En fait, ces Lorenzacio comme ces Brutus ont un amour fétichiste de la vérité - , on ne sait pas ce qu'ils veulent en faire, et on s'aperçoit qu'ils aiment la vérité comme ils s'aiment eux-mêmes, c'est-à-dire très fort, comme s'ils se croyaient vrais, intégralement vrais; ce qui est le début de la morbidité. Donc, dans leur camp il y a déjà la mort - et Shakespeare nous le montre génialement; il sait user des démons, des esprits, des sorcières, des spectres, comme d'un clavier très souple; et là, dans le camp de Brutus, il y aura Jules César présent en forme de spectre, qui révèlera à Brutus sa posture morbide en lui dictant de porter sur lui la mort qu'il n'a pas réussi à donner avec assez d'amour pour la vie. S'il aimait vraiment la liberté, il aurait dû honorer cet amour en se laissant prendre à cette place de souveraineté, en acceptant que le peuple le mette là. Or il a fui cette place. Et cela préfigure la fin de la bataille de Philippes, où les deux conjurés Cassius et Brutus mourront de la même mort en retournant sur eux l'épée. Cela confirme que c'était bien un meurtre narcissique qu'ils avaient commis, c'est-à-dire qu'en tuant le tyran ils tuaient ce qu'en eux-mêmes ils ne supportaient pas, l'ombre d'eux-mêmes; ils l'ont atteinte dans une sorte d'étreinte morbide avec le père; étreinte narcissique, assez homosexuelle; mais laissons ces détails-là, la rigueur de la pièce les rend presque inutiles. Ce qui apparaît en toute clarté, c'est que la guerre, le 
chaos, le désordre, sont les secousses du destin par quoi le temps, le symbolique, cherche un certain ordre pour s'articuler, pour inscrire un passage en forme d'histoire. C'est banal, aujourd'hui, de dire qu'un ordre peut naître du désordre. On l'illustre par l'expérience où l'on met dans une boîte des dés polarisés c'est-à-dire aimantés, nord/sud: une alternance oui/non, en somme; et on agite l'ensemble: tout le monde s'attend à ce qu'en ouvrant la boîte on trouve le chaos total; mais on trouve des architectures d'une forte régularité; c'est que le désordre n'agit pas sur une matière totalement folle; il porte sur des textures déjà polarisées. Il agit comme fécondant sur une première complexité pour en produire une autre. Et c'est, me semble-t-il, une des fonctions de la guerre; c'est patent dans les tétralogies. Dans Henri VI, il est clair que ce n'est pas la mainmise sur la souveraineté qui est en jeu, comme dans Jules César; c'est le partage de la souveraineté ou plutôt le partage de la valeur, sa répartition «équilibrée »... Cette guerre des Roses commence par l'affrontement de deux camps, affrontement très dur où chaque camp semble dire presque textuellement: Reconnaissez que je vaux mieux que vous! Et l'autre rétorque de même: C'est à vous de reconnaître que je vaux mieux, que ma valeur vaut mieux que la vôtre. C'est l'impossible partage de la valeur au-delà duquel se profile l'impossible fondation de la valeur. Quelle est la valeur? qu'est-ce qui fonde la valeur? Elle se fonde toujours après-coup; ça aura été de la valeur, quand l'épreuve violente, guerrière, apporte l'issue qui honore une certaine justesse des tensions en jeu, des processus en cours. Voyez cette scène extraordinaire et pathétique où Talbot et son fils se retrouvent encerclés; Shakespeare ne met pas des scènes comme celle-là au hasard; de même, il ne met pas au hasard une scène comme celle où le roi Henri VI fait la chasse à SaintAlbans et voit arriver un type qui crie «miracle» car il a retrouvé la vue. Il se dit aveugle de naissance, et l'escroquerie se démonte dans la scène où Gloucester lui demande: «quelle est la couleur de mon vêtement?», et l'autre répond: «Rouge, rouge sang»; «et la couleur de 
cet autre vêtement?» «Noir, noir comme le geai». «Et quel est mon nom?» Ça, il ne le sait pas. Et il se révèle escroc car s'il avait été aveugle de naissance il saurait distinguer les couleurs mais pas les nommer. Tout cela n'est pas mis au hasard, c'est bien la question du rapport entre le nom, entité symbolique, et la pure réalité, la différence perçue, réalité immédiate. La scène où Talbot et son fils sont encerclés, et vont mourir pose aussi la question d'une impasse de la transmission - paternité et filiation - dans un contexte totalement livré à l'abrutissement narcissique puisqu'on a là deux armées anglaises commandées par deux chefs qui se détestent et dont aucun ne veut être le premier à envoyer les renforts parce que c'est à l'autre de le lui demander. Et c'est dit textuellement; ce n'est pas une sorte de sacrifice ou d'oblation aux dieux de la guerre, c'est la signature d'un niveau de minabilité des parties en présence face à quelque chose d'important qui s'appelle la demande. Aucun des deux ne veut demander; or qu'est-ce que ne pas pouvoir demander? c'est ne pas pouvoir reconnaître ne serait-ce qu'un instant qu'on est en manque de valeur, qu'on n'a pas la valeur suprême. Conséquence: un père et un fils devront mourir en même temps; transmission rendue morbide...

On s'aperçoit que la course à la valeur, notamment la course à la souveraineté, est chaque fois remportée par des gens qui non seulement sauront jouer sur la crispation narcissique de leurs adversaires tout en sachant articuler leurs différents manques de valeur jusqu'à faire surgir, à partir de ces manques, quelque chose qui opérera comme une source de valeur, venue d'ailleurs, en force.

Si cette thématique de la guerre est si forte c'est aussi comme tiraillement entre deux termes jusqu'à ce que le Tiers apparaisse. Il y a toujours du tiers - il peut n'être autre que la mort, mais on veut le faire parler; comme tel. L'autre jour un petit film sur la guerre des Malouines, m'a étonné: il montre la guerre, mais aussi que les soldats, anglais, et argentins, qui se tiraient dessus d'une façon ultramoderne, révélaient leur tiraillement comme surplombés par la présence d'une tierce Personne qui était 
la mort. On eût dit qu'ils se battaient non pas les uns contre les autres, mais qu'ils se battaient l'un et l'autre contre la Mort. C'est dire que dans la guerre il n'y a pas que ce tiraillement des deux ensembles inconciliables; il y leur mise à tous les deux au regard du tiers qui peut être la mort, mais qui peut être la Loi, la légitimité, c'est-à-dire la transmission de la Loi. Il y a d'ailleurs différents niveaux de légitimité qui exigent pour venir au monde, pour venir au jour, une épreuve douloureuse d'accouchement; où quand les rouges sont au pouvoir, les blancs viennent hurler à l'escroquerie: «vous n'êtes pas légitimes!» $\mathrm{Et}$ inversement. Ils ont tous deux raison et tort car vu l'effet de transmission de génération, on est devant des questions du genre: «qu'est-ce qu'une illégitimité si elle a réussi à passer plusieurs générations par de la légitimité?» Autrement dit on assiste à une lutte, symbolique aussi et donc aussi charnelle et corporelle, entre des légimités qui s'entrecoupent. Et l'on voit que le problème n'est pas de savoir lequel «vaut mieux que l'autre», mais lequel peut mieux inscrire au regard du Tiers sa proximité à la «Loi». Chaque fois, le sexe et les générations perturbent le problème et en font un problème de transmission symbolique. Or l'épreuve est dans le vide intrinsèque à la loi, dans l'absence d'une instance absolue qui dirait «c'est vous les meilleurs et pas eux». Cette instance serait affolante si elle existait vraiment; son vide est vivifiant tout autant que meurtrier. Comment s'y prennent les humains pour inventer un germe de valeur capable de se reproduire et de prendre assez de force pour se renforcer encore à partir de désordres et des chocs, et se nourrir à partir de ces épreuves mêmes?

Henri VI monte et démonte la chose : c'est un espace formidable, car la pièce commence quand Henri VI a neuf mois, ce qui est un âge intéressant, une sorte de contre-conception, de souveraineté contraceptive si je puis dire; et la pièce finit quand il est poignardé par le futur Richard III. On a devant nous un personnage particulier, dont les exégèses ont retenu certains traits: tiens, c'est bizarre, il est dévot... ou : c'est curieux, il est faible et 
un peu trop sensible au charme d'une femme... En fait Shakespeare nous a bricolé le personnage avec une rigueur inouïe; il nous campe un être qui ne supporte pas d'avoir devant lui le conflit. Or les trois pièces vont se dérouler dans un conflit permanent; on se demande alors quelle en est la portée. Et pourquoi par exemple Henri VI est-il dévot? On n'a pas dit un seul mot de sa mère, dans la pièce; pas un mot de son père si ce n'est qu'on est en train de l'enterrer au tout début. Et voilà un être qui a grandi sous l'autorité quasi perverse du Protecteur, c'est-à-dire, sous une autorité qui se réclame de lui pour être autoritaire. Il faut avoir une sacrée carrure, déjà en place, pour supporter un tel champ de forces. Cet homme n'a aucun rapport possible à l'affrontement; et lorsqu'il se trouve devant le conflit naissant des deux Roses, devant ces deux camps qui sont en train de faire les guignols chacun avec sa rose, il ne s'aperçoit pas que lui, très lointain, très gentil (c'est-à-dire échappant à toute trace de conflit), il ne s'aperçoit même pas qu'il a déjà choisi puisqu'il prend une rose...; et rouge. C'est étonnant comme il a l'art de trancher, ce brave souverain - il leur dit: mais pour moi vous êtes tous pareils! rouge ou blanc c'est tout comme! Il leur dit en substance: d'ailleurs si je prends une rose - et comme par hasard il prend la rouge - et que je la porte là, qu'est-ce que ça change?... Du coup le camp de la rose blanche est en train de blanchir de rage... Il a donc choisi à son insu, cet homme qui fuit le conflit; c'est dire qu'il n'a aucun sens de l'accent symbolique de ses actes parce qu'il n'a aucun rapport à ce qu'est un conflit en tant que source et support de vie.

On dirait que la guerre est meurtrière parce que le conflit vivant et source de vie n'a pas trouvé entendeur; n'a trouvé personne pour recueillir ces brins de vie; alors ça va passer en négatif, ça se décidera selon le bon vouloir de la mort, c'est-à-dire selon la manière dont chacun pourra jouer sa partie avec ce terme étonnant de la vie qui s'appelle la mort, et qui est toujours à relancer, puisqu'il porte la trace des transmissions de loi et de non-loi.

Je m'arrête là pour recevoir vos questions. Juste un 
mot, puisque j'ai annoncé que j'en dirai un sur Macbeth. Là c'est encore plus vrai et plus violent encore parce que l'effet Macbeth touche au rapport entre la parole et l'acte en tant que l'un des deux termes (mais lequel?) authentifie l'autre. Ainsi on dit à Macbeth deux ou trois petites choses «vraies» - du genre: vous vous appelez bien Cawdor, n'est-ce pas? et vous avez bien une maison à trente bornes d'ici, exact?...- des choses qui le capturent dans la vérité, puis on lui assène une promesse: tu seras roi. Et lui va se poser la question que se pose tout le monde: ce que je fais là, est-ce bien mon désir? si je tue le roi, pour hâter l'accomplissement de mon destin, cela semble illégitime, mais cela ne va-t-il pas dans le sens de la «loi» plus transcendante et plus secrète dont les sorcières seraient l'écho? Il ne supporte pas le vide ou le creux de la Loi, cette part d'insu qui laisse du jeu au désir. Il se fascine sur ce vide qu'il croit combler; et j'ai montré dans mon livre la logique hallucinatoire et hypnotique qui s'ensuit. En tout cas, cette logique le met en guerre avec tout, avec tous, y compris lui-même. Il va craquer devant ce même vide qui revient et qui se fait autour de lui. Et l'issue même de la guerre confirme notre propos: dans la scène finale Macbeth entérine sa défaite, dès lors que le camp d'en face lui apporte le mot, le mot de la fin: la parole de la sorcière s'accomplissant. Comme quoi on ne perd que les guerres qui sont déjà perdues, et on ne gagne que celles qu'il était presque inutile de livrer si l'adversaire ne s'entêtait à se prendre pour la Loi.

Daniel SIBONY

Psychanalyste, écrivain. A publié notamment: Avec Shakespeare (Grasset, 1988) et récemment: Entre dire et faire: penser la technique (Grasset). 